\title{
MIMO-PID Controller For Deregulated environment
}

\author{
Mallem Pradeep Kumar ${ }^{1}$, K. Vimala Kumar ${ }^{2}$ \\ M.Tech Scholar, Department of EEE, JNTUA College of Engineering Pulivendula, India ${ }^{1}$ \\ Assistant Professor, Department of EEE, JNTUA College of Engineering Pulivendula, India ${ }^{2}$
}

\begin{abstract}
This assessment represents a Multi-Input-Multi-Output-Proportional-Integral-Derivative (MIMO-PID) controller for load frequency balance issues. The objective of load frequency control is to minimize the transient deviations in area frequency and tie-line power. To improve the robustness of the system the transfer function $\mathrm{H}_{\infty}$ norm is used. So the proposed technique is known as $\mathrm{PIDH}_{\infty}$ controller. The LFC problem is articulated as a $\mathrm{H}_{\infty} \mathrm{SOF}$ (State Output Feedback) control approach to attain the decentralized robust MIMO-PIDH ${ }_{\infty}$ controller. $\mathrm{H}_{\infty}$ controller is used here for accomplishing stabilization with definite performance. The optimization difficult is formulated and iterative LMI algorithm is used to tune the control parameters of the MIMO-PIDH ${ }_{\infty}$ controller. The simulation results show the proposed strategy is very effective and guaranteed good robust performance against the parametric uncertainties and load changes.
\end{abstract}

Keywords: Hœ control; LMI; Area Control Error; Load Frequency Control; MIMO-PID control.

\section{INTRODUCTION}

In electrical power system due to deregulation there are PID Controller defined as: unrivalled changes in system and theyraise to changes in the competitive market. The role of control in power system (either man-made operation or instinctive) is to shield operation by returning when subjected to disturbances. In other words control in power system means continuing of system within soothed limits of performance to disturbances, such as short circuits and generation loss or load loss.Lot of study is carried out and on work about this LFC in restructured power system [1][8]. With vibrant and bilateral contracts researchers have modified AGC conventional one [1]-[3]. New market schemes and their operational tools are proposed in [2]. Modified AGC is presented in [4]. LMI approach with decentralized strategy proposed in [7]. Neuro-fuzzy adaptive control is proposed in [8]. In this research threearea power system is specified as dynamic model. The distribution companies (DISCOs) can contact generation companies (GENCOs) for their power needs with different area contracts under independent service operator (ISO). For contract execution a matrix DISCO participation matrix (DPM) is used for conception [4]. The control problem is formulated as $\mathrm{H} \infty$ static output feedback (SOF) control approach to get novel decentralized MIMO-PID controller based on LMI algorithm. The robustness of proposed controller shown through simulation results.

\section{Proposed Control Strategy}

In this section momentary view of decentralized MIMOPIDHo control through LMI is followed

System model defined as:

$$
\dot{x}=A x+B_{1} w+B_{2} u
$$

$z_{\infty}=C_{1} x+D_{12} u$

$y=C_{2} x$

$$
u=K_{1} y+K_{2} \int_{0}^{t} y d t+K_{3} \frac{d y}{d t}
$$

Here K1,K2,K3 matrices are to be designed (PID Gains) Output feedback $\mathrm{H} \infty$-control problem is to find controller of

$$
u=K y
$$

From above closed loop transfer function of infinite-form from $\mathrm{Z} \infty$ to $\mathrm{w}$

$\left(\left\|T_{z_{\infty}{ }^{w}}\right\|\right)<\gamma$

Consider $z_{1}=x \quad z_{2}=\int_{0}^{t} y d t \quad$ and $\quad z=\left[\begin{array}{c}z 1 \\ z 2\end{array}\right]$

The variable as viewed state vector of new system

$\dot{z}_{1}=\dot{x}=A z_{1}+B_{1} w+B_{2} u$

$\dot{z}_{2}=C_{2} z_{1}$

$z_{1}=x=C_{1} x+D_{12} u$

PID problem is reduced through static output feedback (SOF) control system as

$\dot{z}=\bar{A} z+\overline{B_{1}} w+\overline{B_{2}} u$

$\bar{z}_{\infty}=\bar{C}_{1} z+\overline{D_{12}} u$

$\bar{y}=\overline{C_{2}} z+\overline{D_{21}} w$

$u=\bar{K} \bar{y}$

Where

$\bar{A}=\left[\begin{array}{cc}A & 0 \\ C_{2} & 0\end{array}\right], \overline{B_{1}}=\left[\begin{array}{c}B_{1} \\ 0\end{array}\right], \overline{B_{2}}=\left[\begin{array}{c}B_{2} \\ 0\end{array}\right]$,

$\overline{C_{1}}=\left[\begin{array}{ll}C_{1} & 0\end{array}\right], \overline{C_{2}}=\left[\begin{array}{lll}\overline{C_{21}} & \overline{C_{22}} & \overline{C_{23}}\end{array}\right]^{T}$,

$\overline{D_{12}}=D_{12}, \overline{D_{21}}=\left[\begin{array}{lll}0 & 0 & C_{2} B_{1}\end{array}\right]^{T}$
And $\bar{K}=\left[\begin{array}{lll}\overline{K_{1}} & \overline{K_{2}} & \overline{K_{3}}\end{array}\right]$ 
Once $\bar{K}$ is found original PID gains can be obtained

$K_{3}=\overline{K_{3}}\left(I+C_{2} B_{2} \overline{K_{3}}\right)^{-1}$,

$K_{2}=\left(I-K_{3} C_{2} B_{2}\right) \overline{K_{2}}$

$K_{1}=\left(I-K_{3} C_{2} B_{2}\right) \overline{K_{1}}$

LMI algorithm for $P I D H$ optimizing system in equation

(4) follows

Step 1: From state space model in (1)

Compute $\bar{A}, \overline{B_{1}}, \overline{B_{2}}, \overline{C_{1}}$ and $\overline{C_{2}}$ by using (7)

and select performance index $(\gamma)$

Step 2: $\quad$ Select $\mathrm{Q}>0$ and solve for riccati equation

$$
\bar{A}^{T} P+P \bar{A}-P{\overline{B_{2}}}_{B_{2}}^{T} P+Q=0, P>0
$$

Set $i=1$ and $X_{i}=P$

Step 3: $\quad$ solve optimization problem for $P_{i}, \bar{K}$ and $a_{i}$ Optimization 1: minimize $a_{i}$ subject to LMI constraints

$\left[\begin{array}{cccc}\sum_{\bar{B}_{1}}{ }^{T} P_{i} & P_{i} \overline{B_{1}} & \left(\overline{C_{1}}+\overline{D_{12}} \overline{C_{C}}\right)^{T} & \left(\bar{B}_{2}{ }^{T} P_{i}+\bar{K} \overline{C_{2}}\right)^{T} \\ \overline{C_{1}}+\overline{D_{12}} \bar{K} \bar{C}_{2} & 0 & 0 & 0 \\ \bar{B}_{2}{ }^{T} P_{i}+\bar{K} \bar{C}_{2} & 0 & 0 & -\gamma\end{array}\right]<0$

Where $\sum=\bar{A}^{T} P_{i}+P_{i} \bar{A}-X_{i}{\overline{B_{2}}}_{B_{2}}{ }^{T} P_{i}-P_{i}{\overline{B_{2}}}_{B_{2}}{ }^{T} X_{i}+X_{i}{\overline{B_{2}}}_{B_{2}}{ }^{T} X_{i}-a P_{i}$ $a_{i}^{*}$ Denotes minimized value of $a_{i}$

Step 4: If $a_{i} \leq 0$ the matrix pair $\left(P_{i}, \bar{K}\right)$ solves the problem. Stop. Otherwise go to step 5

Step 5: solve optimization problem for $P_{i}$ and $\bar{K}$

Optimization 2: minimize $P_{i}$ subject to LMI constraint in (10) with $a_{i}=a_{i}^{*}$.

Optimal $P_{i}$ denoted by $P_{i}^{*}$

Step 6: If $\left\|X \overline{B_{1}}-P_{i}^{*} \overline{B_{1}}\right\|<\in$ go to step 7 otherwise set $i=i+1, X_{i}=P_{i}^{*}$ and go to step.

Here $\in$ denotes tolerance (prescribed)

Step 7: If obtained $\bar{K}$ satisfies gain constant, it is desirable otherwise change weight $n_{i}, Q$ and $\gamma$ go to step 1.

\section{III.DESIGN MODEL OF CONTROLLER}

Projected model for three area power system with interconnections in deregulated background as [4] and [8] is shown in Fig 1.

State space model as follows:

$$
\begin{array}{ll}
\dot{x}=A x+B_{1} w+B_{2} u & \\
\mathrm{P}(\mathrm{s}): & z_{\infty}=C_{1} x+D_{12} u
\end{array}
$$

$y=C_{2} x+D_{21} w$

Here

$$
x=\left[\begin{array}{lllllllll}
\Delta X_{E 1} & \Delta P_{t 1} & \Delta f_{1} & \Delta X_{E 2} & \Delta P_{t 2} & \Delta P_{\text {tiel2 }} & \Delta X_{E 3} & \Delta P_{t 3} & \Delta f_{2}
\end{array}\right.
$$

$\left.\Delta X_{E 4} \quad \Delta P_{t 4} \quad \Delta P_{t i e 23} \quad \Delta X_{E 5} \quad \Delta P_{t 5} \quad \Delta f_{3} \quad \Delta X_{E 6} \quad \Delta P_{t 6} \quad \Delta P_{\text {tie31 }}\right]^{T}$

$w=\left[\begin{array}{llllll}\Delta P_{L 1} & \Delta P_{L 2} & \Delta P_{L 3} & \Delta P_{L 4} & \Delta P_{L 5} & \Delta P_{L 6}\end{array}\right]^{T}$

$u=\left[\begin{array}{lll}u_{1} & u_{2} & u_{3}\end{array}\right]^{T}$ $z_{\infty}$ is a controlled output vector

$y=\left[\begin{array}{lllll}A C E_{1} & A C E_{2} & A C E_{3} & \Delta f_{1} & \Delta f_{2}\end{array}\right.$

$$
\left.\Delta f_{3} \quad \Delta P_{\text {tie } 12} \quad \Delta P_{\text {tie } 23} \quad \Delta P_{\text {tie } 31}\right]^{T}
$$

$\mathrm{y}$ is a measured output vector

Controlled output vector considered as follows

$$
\begin{gathered}
z_{\infty}=\left[\begin{array}{ccccc}
n_{1} \Delta f_{1} & n_{2} \Delta f_{2} & n_{3} \Delta f_{3} & n_{4} \Delta P_{\text {tie } 12} & n_{5} \Delta P_{\text {tie } 23} \\
n_{6} \Delta P_{\text {tie } 31} & n_{7} u_{1} & n_{8} u_{2} & n_{9} u_{3}
\end{array}\right]^{T} \text { (12) }
\end{gathered}
$$

Here $n_{1}$ to $n_{9}$ are constants and weight coefficients are chosen by designer.

Consider three area power system parameters in Fig 1. as $\mathrm{K}_{\mathrm{g}}=1.0, \quad \mathrm{~T}_{\mathrm{g}}=0.08 \mathrm{~s}, \quad \mathrm{~K}_{\mathrm{t}}=1.0, \quad \mathrm{~T}_{\mathrm{t}}=0.3 \mathrm{~s}, \quad \mathrm{R}_{\mathrm{i}}=2.4(\mathrm{~Hz} / \mathrm{pu})$, $\mathrm{B}_{\mathrm{i}}=0.425(\mathrm{pu} / \mathrm{Hz}) \quad \mathrm{K}_{\mathrm{P}}=120(\mathrm{pu} / \mathrm{Hz}), \mathrm{Tp}=20 \mathrm{~s}, \mathrm{~T}_{12}=0.0868 \mathrm{~s}$, $\mathrm{T}_{23}=0.0868 \mathrm{~s}, \mathrm{~T}_{31}=0.0868 \mathrm{~s}$
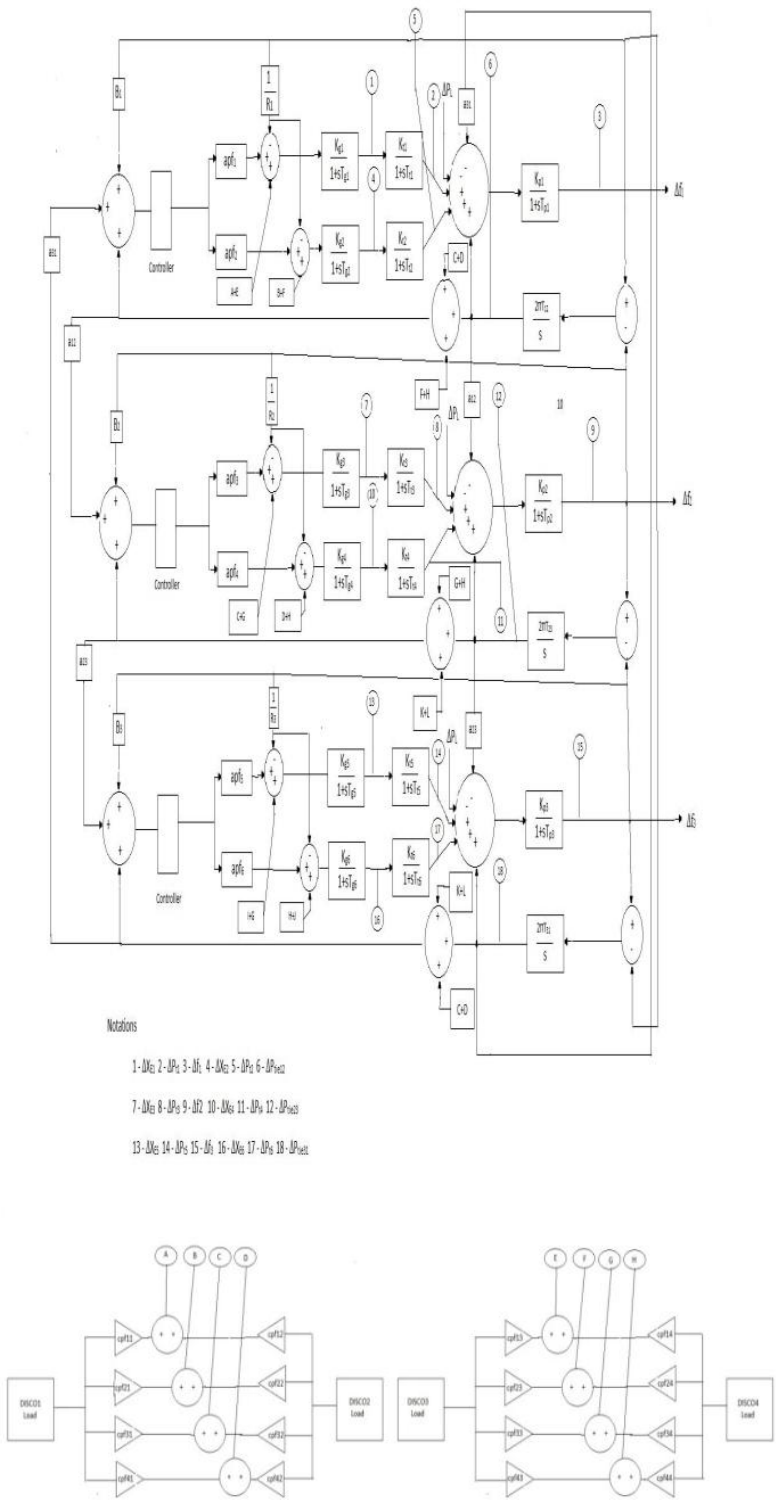
INTERNATIONAL JOURNAL OF INNOVATIVE RESEARCH IN ELECTRICAL, ELECTRONICS, INSTRUMENTATION AND CONTROL ENGINEERING Vol. 2, Issue 12, December 2014

Figure1. Three area power system with cpfs.

\section{IV.RESULTS}

Free contracts transactions based scenario:

In this section all DISCOs are contracted with GENCOs as per DPM (Dynamic Participation Factor).

$$
D P M=\left[\begin{array}{cccccc}
0.5 & 0.25 & 0 & 0.3 & 0.8 & 0 \\
0.2 & 0.25 & 0 & 0 & 0.1 & 0.1 \\
0 & 0.25 & 1 & 0.7 & 0.1 & 0.1 \\
0.3 & 0.25 & 0 & 0 & 0 & 0.8
\end{array}\right]
$$

All GENCOs in each area participate as follows

$$
\begin{array}{ll}
\text { apf } 1=0.50 & \text { apf } 2=1-\text { apf } 1=0.50 \\
\text { apf3 }=0.50 & \text { apf } 4=1-\text { apf } 3=0.50 \\
\text { apf5 }=0.50 & \text { apf6 }=1-\text { apf } 5=0.50
\end{array}
$$

Load variations considered as

$$
\begin{array}{lll}
\Delta \mathrm{P}_{\mathrm{L} 1}=0.75 \mathrm{pu} & \Delta \mathrm{P}_{\mathrm{L} 2}=0.75 \mathrm{pu} & \Delta \mathrm{P}_{\mathrm{L} 3}=0.75 \mathrm{pu} \\
\Delta \mathrm{P}_{\mathrm{L} 4}=0.75 \mathrm{pu} & \Delta \mathrm{P}_{\mathrm{L} 5}=0.75 \mathrm{pu} & \Delta \mathrm{P}_{\mathrm{L} 6}=0.75 \mathrm{pu}
\end{array}
$$

$\Delta \mathrm{P}_{\mathrm{M} 1}=0.5 * 0.75+0.25 * 0.75+0 *(0.75)+0.3 *(0.75)+0 *(0.75)$

$+0 *(0.75)=0.7875 \mathrm{pu}$

Correspondingly

$\Delta \mathrm{P}_{\mathrm{M} 2}=0.3375 \mathrm{pu} \quad \Delta \mathrm{P}_{\mathrm{M} 3}=0.4275 \mathrm{pu} \quad \Delta \mathrm{P}_{\mathrm{M} 4}=0.4125 \mathrm{pu}$

$\Delta \mathrm{P}_{\mathrm{M} 5}=0.5475 \mathrm{pu} \quad \Delta \mathrm{P}_{\mathrm{M} 6}=0.1500 \mathrm{pu}$

Power generated from GENCOs in area-I, II and III shown in Fig 3. With desired values of $\Delta \mathrm{P}_{\mathrm{Mi}}$. Test results show robustness of the system.
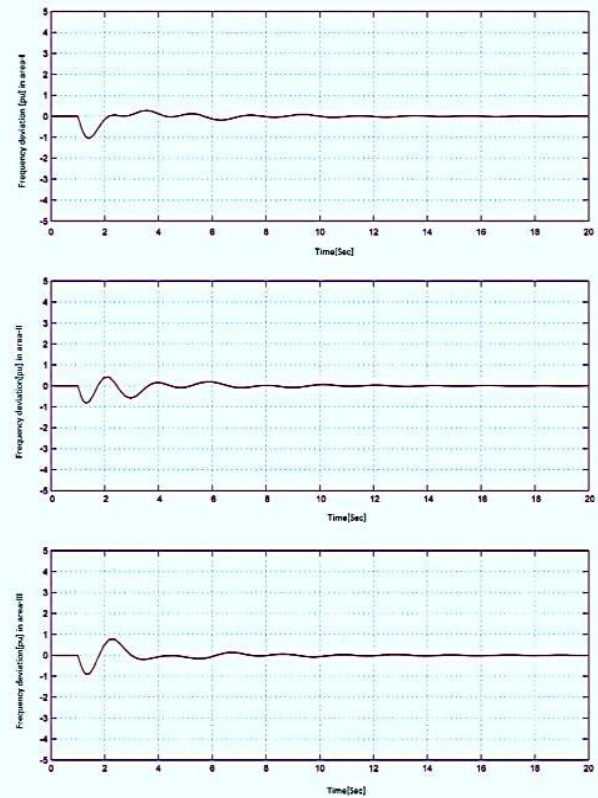

Figure2. Frequency deviations in corresponding areas
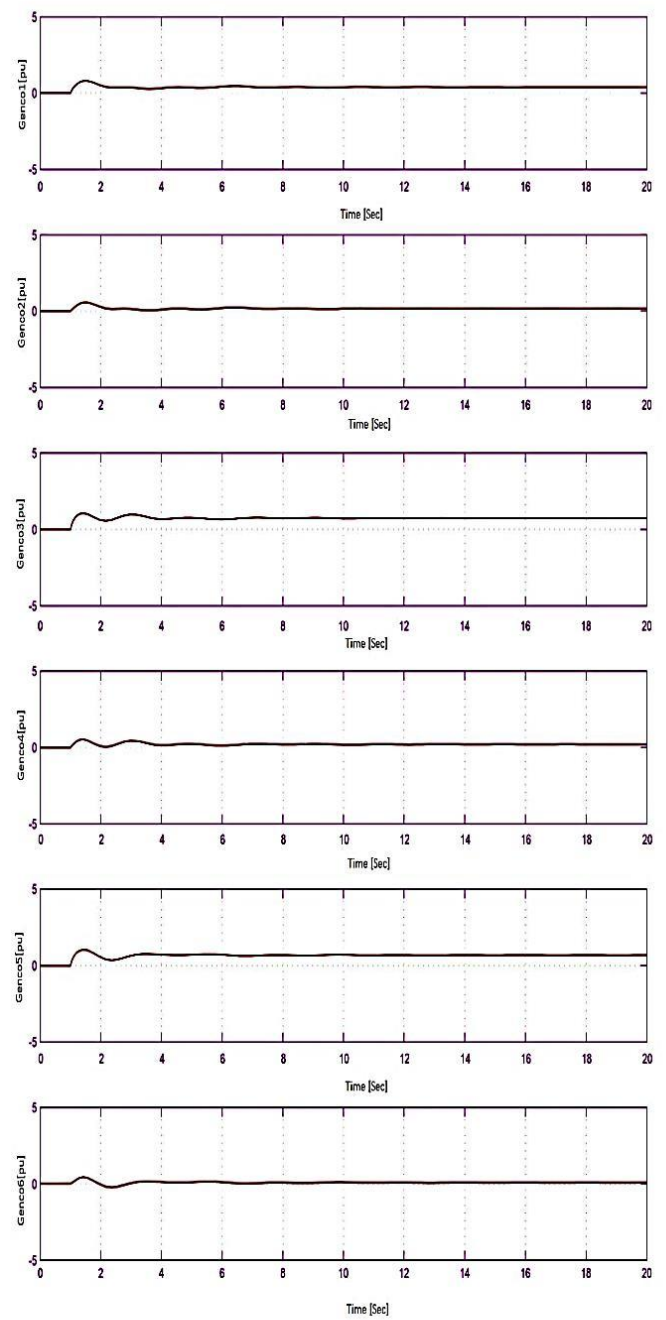

Time offiset 0

Figure3. Generated Power

\section{Conclusion}

In three area power system with reheat type thermal power stations this researchoffers MIMO-PID decentralized controller in restructured power system. To diminish effects of uncontrolled changes in system in area's inside loads and in interconnected loads control actions are calculated through LMI. For guaranteed performance and robustness this controller is effective and efficient against variations in loads and parametric uncertainties.

\section{ACKNOWLEDGMENT}

I thank god for giving good knowledge and health and thank my parents for their love towards my studies and my guide K. Vimala Kumar for continuing cooperation in this research.

\section{REFERENCES}

[1] R.D.Christie, and A.Bose, "Load Frequency control issues in power systems operation after deregulation" IEEE Trans. Power Systems, vol. 11, no. 3, pp. 1191-1200, Aug. 1996. 
[2] J. Kumar, Kah-Hoe Ng, and G. Sheble, "AGC Simulator for price based operation part 1:a model" IEEE Trans. Power Systems, vol. 12, no. 2, pp. 527-532, May 1997.

[3] J. Kumar, Kah-Hoe Ng, and G. Sheble, "AGC Simulator for price based operation part II:case study results" IEEE Trans. Power Systems, vol. 12, no. 2, pp. 523-538, May 1997.

[4] V.Donde, M.A.Pai, and I.A.Hiskens, "Simulation and optimization after deregulation" IEEE Trans. Power Systems, vol. 16, no. 3, pp. 481-489, Aug. 2001.

[5] D.Rerkpreedapong, A. Hasanovic, and A.Feliachi, "Robust load frequency control using genetic algorithm and linear matrix inequalities", IEEE Trans. Power Syst., vol. 18, no. 2, pp. 855-861.

[6] H. Bewrani, Y. Mitani, and K. Tsuji, "Robust decentralized load frequency control using an iterative linear matrix inequalities algorithm", IEE Proc. Gener. Trans. Distrib. Vol.151, no. 3, pp. 347-354, 2004.

[7] B.Tyagi and S. C. Srivastava, "A decentralized automatic generation control scheme for competitive electricity markets," IEEE Trans. Power System, vol. 21, no. 1, pp. 312-320, 2006.

[8] S. H. Hosseini, and, A. H. Etemadi, "Adaptive neuro-fizzy inference system based automatic generation control," Elsevier, Electric Power Systems Research vol. 78, pp. 1230-1239, 2008.

[9] Nilay Kumar. N. Shah, Chetan D. Kotwal, "The State Space Modeling of Single, Two and Three ALFC of Power System Using Integral Control and Optimal LQR Control Method," IOSR Journal of Engineering, Vol. 2(3) pp: 501-510, March 2012.

[10] Ahmed Bensenouci, A. M. Abdel Ghany, "Performance Analysis and Comparative Study of LMI-Based Iterative PID LoadFrequency Controllers of Single-Area Power System," WSEAS Trans. On. Power Systems, issue no. 2, pp. 85-97, April 2010.

\section{BIOGRAPHIES}

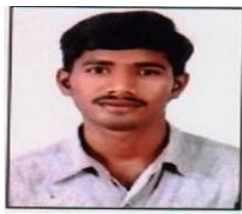

Mallem Pradeep Kumar is pursuing Master of Technology in Electrical Power Systems Engineering from JNTUA College of Engineering Pulivendula-516390, Andhra Pradesh, India. He is presently working on his project under the guidance of Assistant Professor K.Vimala Kumar.

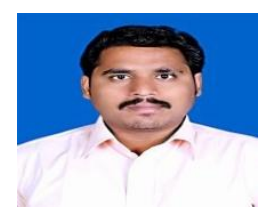

K.Vimala Kumar received the Master of Technology degree in Electrical \& Electronics Engineering from Jawaharlal Nehru Technological University Hyderabad, India 2008. He is a research student of Jawaharlal Nehru Technological University, Anantapur, India. Currently he is working as Assistant Professor in the department of Electrical and Electronics Engineering, J.N.T.U.A College of Engineering, Pulivendula-516390, Andhra Pradesh, India. His interest areas are power system deregulation, power system operation and control, power system design and dynamic load modelling. 